\title{
Intensity modulated radiotherapy induces pro-inflammatory and pro-survival responses in prostate cancer patients
}

\author{
HOUSSEIN EL-SAGHIRE ${ }^{1,2}$, CHARLOT VANDEVOORDE ${ }^{2}$, PIET OST ${ }^{4}$, PIETER MONSIEURS ${ }^{1}$, \\ ARLETTE MICHAUX $^{1}$, GERT DE MEERLEER ${ }^{4}$, SARAH BAATOUT $^{1,3}$ and HUBERT THIERENS ${ }^{2}$ \\ ${ }^{1}$ Radiobiology Unit, Molecular and Cellular Biology, Belgian Nuclear Research Centre (SCK·CEN), Mol; \\ Departments of ${ }^{2}$ Basic Medical Sciences and ${ }^{3}$ Molecular Biotechnology, Ghent University; \\ ${ }^{4}$ Department of Radiation Oncology, Ghent University Hospital, Gent, Belgium
}

Received October 8, 2013; Accepted November 11, 2013

DOI: $10.3892 /$ ijo.2014.2260

\begin{abstract}
Intensity modulated radiotherapy (IMRT) is one of the modern conformal radiotherapies that is widely used within the context of cancer patient treatment. It uses multiple radiation beams targeted to the tumor, however, large volumes of the body receive low doses of irradiation. Using $\gamma-\mathrm{H} 2 \mathrm{AX}$ and global genome expression analysis, we studied the biological responses induced by low doses of ionizing radiation in prostate cancer patients following IMRT. By means of different bioinformatics analyses, we report that IMRT induced an inflammatory response via the induction of viral, adaptive, and innate immune signaling. In response to growth factors and immune-stimulatory signaling, positive regulation in the progression of cell cycle and DNA replication were induced. This denotes pro-inflammatory and pro-survival responses. Furthermore, double strand DNA breaks were induced in every patient $30 \mathrm{~min}$ after the treatment and remaining DNA repair and damage signaling continued after 18-24 h. Nine genes belonging to inflammatory responses (TLR3, SH2DIA and IL18), cell cycle progression (ORC4, SMC2 and CCDC99) and DNA damage and repair ( $R A D 17, S M C 6$ and $M R E 11 A)$ were confirmed by quantitative RT-PCR. This study emphasizes that the risk assessment of health effects from the out-of-field low doses during IMRT should be of concern, as these may increase the risk of secondary cancers and/or systemic inflammation.
\end{abstract}

\section{Introduction}

Currently, intensity modulated radiotherapy (IMRT) is a widely applied conformal radiotherapy modality; in contrast to conventional radiotherapy, IMRT uses multiple beams

Correspondence to: Dr Houssein El-Saghire, Radiobiology Unit, Molecular and Cellular Biology, Belgian Nuclear Research Centre (SCK·CEN), Boeretang 200, $2400 \mathrm{Mol}$, Belgium

E-mail: hesaghir@sckcen.be

Key words: IMRT, low dose, ionizing radiation, inflammatory response, prostate cancer, radiotherapy, microarray, $\gamma$-H2AX across the target field of treatment. This reduces the volume of tissues receiving high doses, but a greater volume of normal tissues still receives low doses of radiation (1-3). It is estimated that IMRT can contribute to $1.5 \%$ increased risk of secondary cancers by 10 years following treatment (4). However, these figures were considered to be overestimated because the calculations of these risks were based on the long-term data obtained from the follow-up of atomic bomb survivors. This population was exposed to a single whole body dose, while IMRT patients receive fractionated doses to specific body parts (5). Besides, other studies moderated the therapeutic effect of IMRT over its potential health side effects $(6,7)$.

Microarrays and DNA damage studies, through measuring the Ser 139 phosphorylated form of histone H2AX $(\gamma-\mathrm{H} 2 \mathrm{AX})$, are emerging applications in the field of radiation biology and biodosimetry. Gene expression studies improved the knowledge on cellular responses to both high and low radiation doses (8-11). On the other hand, $\gamma-\mathrm{H} 2 \mathrm{AX}$ foci immunodetection has been described as useful quantitative biomarker of human lowlevel radiation exposure (12).

In this study, we address the question of understanding the whole blood tissue biological responses in prostate cancer patients receiving low doses of ionizing radiation during IMRT. It is the first study that combines DNA damage and microarray investigations on whole blood samples collected in vivo from patients receiving low doses over a large part of the body. It highlights the mechanisms and the possible health effects involved in response to low doses of ionizing radiation. For the DNA damage assessment $\gamma-\mathrm{H} 2 \mathrm{AX}$ foci were scored. For the analysis of the microarray data, we applied a holistic approach, namely Gene Set Enrichment Analysis (GSEA) that it is known to overcome many of the limitations in individual gene pathway analysis, discussed thoroughly by Subramanian and colleagues (13). In addition, we used differentially expressed genes for Exploratory Gene Association Networks (EGAN) analysis.

\section{Materials and methods}

Patients and sample collection. The study population consisted of 8 prostate cancer patients treated with step and shootIMRT (ss-IMRT) (Elekta Synergy linear accelerator) at the 
Department of Radiation Oncology (Ghent University Hospital, Belgium) between March and May 2013. A dose per fraction to the tumor was $2.09 \mathrm{~Gy}$. After obtaining written approval of the ethics committee at Ghent University Hospital and signed informed consent, blood samples were taken at different timepoints. Blood sampling for the $\gamma-\mathrm{H} 2 \mathrm{AX}$ foci was performed in heparin vacutainer tubes before and $30 \mathrm{~min}$ after the first fraction, blood sampling for the whole genome expression analysis was performed in EDTA vacutainer tubes before the first and second fraction, 18-24 $\mathrm{h}$ after the first fraction.

Dose calculation. The equivalent total body blood dose (DETB) was calculated for each patient based on the treatment planning data. To this end, the mean dose within the skin contour of the scanned volume was normalised to the patient mass. As liver, heart/large blood vessels and lungs contain together $38.5 \%$ of the total blood volume it was assumed that $61.5 \%$ of the blood pool is distributed uniformly over the rest of the body.

$\gamma$-H2AX scoring. The procedure for the $\gamma-\mathrm{H} 2 \mathrm{AX}$ foci assay on T-lymphocytes is described in detail in a previous report (14). Foci analysis was performed with the Cytovision v.2.8 Software 2002 (Applied Imaging, USA) and an Olympus BX60 fluorescent microscope was used with a 100x/1.30 oil lens. Several images of one slide were captured with a digital camera (Applied Imaging), $10 \mathrm{Z}$-stacks with $1.03 \mu \mathrm{m}$ spacing was used.

RNA isolation for microarray gene expression studies. Collected venous blood (4-ml/time-point) was passed through a LeukoLOCK ${ }^{\mathrm{TM}}$ filter (Life Technologies, USA), washed with PBS and leukocytes were stabilized with RNAlater ${ }^{\circledR}$. Filters were capped and stored at $-20^{\circ} \mathrm{C}$. RNA was isolated using LeukoLOCK ${ }^{\mathrm{TM}}$ Isolation System (Life Technologies) according to the manufacturer's instructions. RNA was quantified using a Nanodrop 2000 (Thermo Scientific, USA) spectrophotometer, the quality was assessed with Agilent 2100 Bioanalyzer. All RNA samples had $\geq 8.5$ as an integrity number.

Microarray assay. Using the Ambion ${ }^{\circledR}$ WT Expression kit (Ambion, USA), cDNA was prepared from $10 \mu \mathrm{g}$ of purified cRNA, originally synthesized and purified from $0.25 \mu \mathrm{g}$ of total RNA, following the manufacturer's instructions. The cDNA $(2.75 \mu \mathrm{g})$ was used for fragmentation and labeling using GeneChip ${ }^{\circledR}$ Terminal Labeling kit (Affymetrix, USA). Using GeneChip ${ }^{\circledR}$ Hybridization, Wash and Stain kit (hybridization module) (Affymetrix), and hybridization controls (Affymetrix), fragmented and labeled cDNA was hybridized to Human Gene 1.0 ST arrays (Affymetrix). After hybridization with rotation for $16 \mathrm{~h}$ at $45^{\circ} \mathrm{C}$, arrays were washed and stained, according to the manufacturer's instructions, using GeneChip ${ }^{\circledR}$ Hybridization, Wash and Stain kit (stain module) (Affymetrix). Finally, arrays were scanned immediately using Affymetrix GeneChip ${ }^{\circledR}$ Scanner.

Microarray data processing. Raw Affymetrix data were preprocessed using Partek Genomics Suite v6.6 (Partek Inc.,
USA). Briefly, Robust Multichip Average (RMA) was used for background correction followed by quantile normalization and summarization of multiple probe intensities for each probeset using the median polish approach (15). Gene expression values were obtained by the one-step Tukey method.

Functional analysis - GSEA. GSEA calculates an enrichment score (ES) reflecting the overrepresentation of a certain gene set at the top or bottom of a ranked list of genes found in the expression dataset of two classes. This method applies the Kolmogorov-Smirnov test to find deviation between two distributions. Information on GSEA was reported previously (13). Briefly, genes are ranked using signal-to-noise ratio. Using Kolmogorov-Smirnov statistics, pre-defined sets of genes are scored and significance is tested by empirical permutation followed by correction for multiple hypotheses. The Reactome database was used as reference background for the implemented analysis. In total, the data were analyzed against 674 gene sets downloaded from the Molecular Signature Database (MSigDB) (http://www.broadinstitute.org/ gsea/msigdb/index.jsp). The GSEA software parameters were set to their default values. The statistical significance of the normalized enrichment score (NES) associated to each gene set was assessed through 1,000 random permutations of the phenotypic labels. FDR (false discovery rate) value $<0.05$ was used as a cut-off value for assessing the statistical significance of the estimates. For gene set networks, we used the Enrichment Map plug-in (16) for Cytoscape Desktop program (http://baderlab.org/Software/EnrichmentMap/). Gene sets with FDR values $<0.05$ and having $\geq 50 \%$ overlapping genes are represented in the network.

Functional analysis - Exploratory Gene Association Networks. To test for differential expression between different irradiated conditions and reference conditions (no irradiation) we used repeated measures ANOVA. Differentially expressed genes were defined with a p-value cutoff with a false discovery rate of $<0.05$. Differentially expressed genes were analyzed using Exploratory Gene Association Networks (EGAN, The Regents of the University of California) software to determine differentially regulated pathways. P-values were corrected using Westfall-Young minP method. P-values $<0.05$ were considered significant. For clearer illustrations, not all genes belonging to each pathway are shown in the figures.

Quantitative RT-PCR validation. For quantitative real-time (RT-PCR) confirmation, we selected nine different genes that were shown to be differentially expressed and contributed to the pathway enrichment of immune signaling, DNA damage and repair and cell cycle progression. Briefly, cDNA was prepared from $0.25 \mu \mathrm{g}$ of total RNA using Ambion ${ }^{\circledR}$ WT Expression kit (Ambion) following the manufacturer's instructions. RT-PCR was performed using TaqMan ${ }^{\circledR}$ Gene Expression assays (Applied Biosystems, USA). Each TaqMan assay was run in duplicate for each diluted cDNA sample using TaqMan ${ }^{\circledR}$ Fast Advanced Master Mix (Applied Biosystems). The reactions were run on ABI 7500 Fast RT-PCR system following the manufacturer's recommended PCR program: $95^{\circ} \mathrm{C}$ for $20 \mathrm{sec}$, followed by 40 cycles of $95^{\circ} \mathrm{C}$ for $3 \mathrm{sec}$ and $60^{\circ} \mathrm{C}$ for $30 \mathrm{sec}$. Relative expression values were calculated 


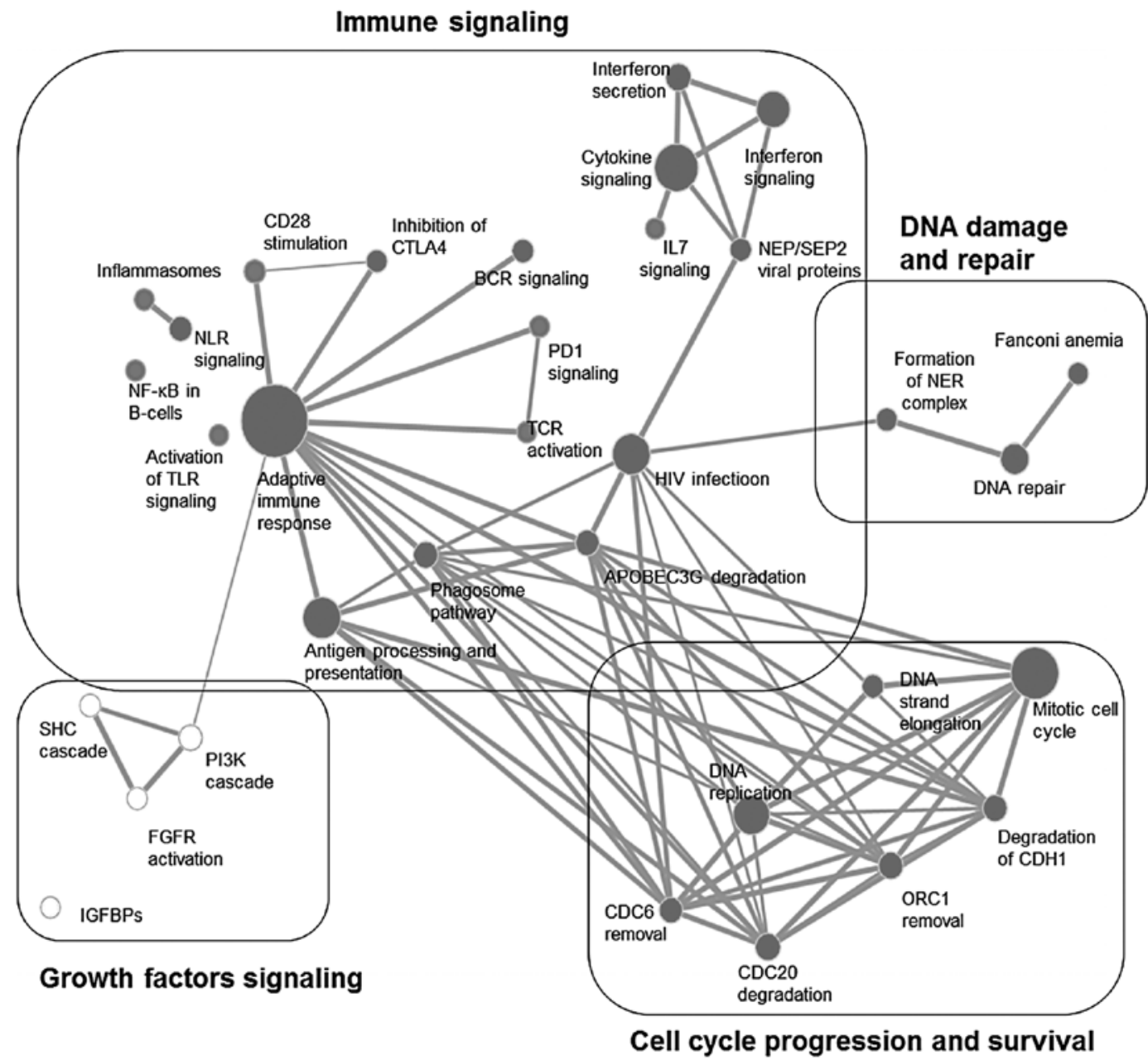

Figure 1. Enrichment map analysis of GSEA results. Each gene set is represented by a node with different size, proportional to the number of genes; the connecting line represents the percentage of overlap and its thickness represents the percentage of overlapping. Black nodes represent upregulated gene sets, whilst white nodes represent downregulated gene sets. A combination of two cut-offs was applied: 5\% FDR and a minimum of 50\% gene overlapping.

by Pfaffl (17) method normalized to $P G K 1$ levels. Relative expression levels were tested for statistical significance using paired t-test, genes having $\mathrm{p}$-values $<0.05$ were considered significant.

\section{Results}

Based on the treatment planning data the equivalent total body dose of one fraction amounted to $30.97 \pm 8.12 \mathrm{mGy}$ (Table III). GSEA enrichment map analysis showed interconnections of 4 different signal transduction categories; these are immune signaling, growth factors signaling, cell cycle progression and survival, as well as DNA damage and repair (Fig. 1). On the other hand, EGAN analysis showed the biological response is divided into three different categories: growth factors and cell cycle progression, viral and immune signaling and metabolism (Table II and Fig. 2).

Low doses of ionizing radiation induces pro-inflammatory response via the activation of viral, adaptive and innate immune signaling. HIV infection and gene sets belonging to the adaptive immune response contributed mainly to the enrichment of the immune signaling cluster (Table I).
The involvement of the viral infection response, along with interferon signaling and secretion and APOBEC3G degradation denotes the induction of an inflammatory response accompanied by DNA damage; the HIV infection node shared a common edge with the DNA damage and repair gene sets (Fig. 1). Furthermore, the enrichment map analysis showed involvement of adaptive immune response activation, particularly CD28 stimulation that works in an opposite way with CTLA4, leading to T-cell receptor activation and cytokine secretion (Table I and Fig. 1). In addition, innate immune gene sets were significantly modulated; these include phagosome pathway, inflammasomes, toll-like receptors and NOD-like receptors (Table I and Fig. 1). Similar to GSEA, EGAN analysis showed the enrichment of signaling involved in viral immune responses. The viral signaling network was composed of several immune-related pathways, namely virus replication, I $\mathrm{B}$ proteins and toll-like receptors. Furthermore, it showed connection with DNA damage and repair node, which is a characteristic of a viral response (Fig. 3 and Table II).

Among the upregulated genes that contributed to the positive regulation of inflammatory response are $S H 2 D 1 A$, TLR3 and IL18 (Figs. 3 and 7A). 
Table I. Statistical significance of GSEA Reactome database gene sets.

\begin{tabular}{|c|c|c|c|}
\hline Category & Gene set & Size of gene set & FDR q-value \\
\hline \multirow[t]{18}{*}{ Immune signaling } & $\mathrm{HIV}^{\mathrm{a}}$ infection & 181 & $<0.0001$ \\
\hline & Interferon secretion & 63 & $<0.0001$ \\
\hline & $\mathrm{NEP}^{\mathrm{b}} / \mathrm{SEP} 2^{\mathrm{c}}$ viral proteins & 25 & $<0.0001$ \\
\hline & Activation of APOE3G degradation via $\mathrm{VIF}^{\mathrm{e}}$ & 47 & 0.001 \\
\hline & $\mathrm{CD} 28^{\mathrm{f}}$ stimulation & 56 & 0.004 \\
\hline & Antigen processing and presentation & 183 & 0.006 \\
\hline & $\mathrm{BCR}^{\mathrm{g}}$ activation & 115 & 0.02 \\
\hline & $\mathrm{TCR}^{\mathrm{h}}$ activation & 13 & 0.009 \\
\hline & Phagosome pathway & 55 & 0.01 \\
\hline & Adaptive immune response & 460 & 0.013 \\
\hline & Inhibition of CTLA $4^{\mathrm{i}}$ & 20 & 0.013 \\
\hline & Inflammasomes & 15 & 0.02 \\
\hline & Activation of $\mathrm{NF} \kappa \mathrm{B}$ & 60 & 0.031 \\
\hline & Activation of TLR ${ }^{\mathrm{j}}$ signaling & 11 & 0.03 \\
\hline & Cytokine signaling & 237 & 0.041 \\
\hline & $\mathrm{NLR}^{\mathrm{k}}$ signaling & 38 & 0.042 \\
\hline & IL7 signaling & 10 & 0.045 \\
\hline & PD1 ${ }^{1}$ signaling & 15 & 0.047 \\
\hline \multirow[t]{7}{*}{ Cell cycle } & Mitotic cell cycle & 278 & $<0.0001$ \\
\hline & Degradation of mitotic proteins via $\mathrm{CDC} 20^{\mathrm{m}}$ & 61 & $<0.0001$ \\
\hline & Degradation of $\mathrm{CDH} 1^{\mathrm{n}}$ & 54 & $<0.0001$ \\
\hline & Removal of $\mathrm{CDC}^{\circ}$ & 45 & $<0.0001$ \\
\hline & DNA replication & 170 & $<0.0001$ \\
\hline & Chromosome maintenance & 100 & 0.028 \\
\hline & ORC $1^{\mathrm{p}}$ removal & 58 & 0.003 \\
\hline \multirow[t]{3}{*}{ DNA damage and repair } & DNA repair & 91 & $<0.0001$ \\
\hline & Formation of NER $^{\mathrm{q}}$ complex & 17 & 0.0009 \\
\hline & Fanconi anemia & 16 & 0.001 \\
\hline \multirow[t]{4}{*}{ Growth signaling } & FGFR $^{\mathrm{r}}$ activation & 21 & $<0.0001$ \\
\hline & SHC $^{\text {s }}$ cascade & 25 & 0.001 \\
\hline & PI3K $\mathrm{K}^{\mathrm{t}}$ cascade & 51 & 0.029 \\
\hline & IGFBPs $^{u}$ & 14 & 0.023 \\
\hline \multirow[t]{5}{*}{ Metabolism } & Amino acids metabolism & 16 & $<0.0001$ \\
\hline & Metabolism of lipids & 19 & 0.001 \\
\hline & Metabolism of proteins & 24 & 0.002 \\
\hline & $\mathrm{TCA}^{\mathrm{v}}$ cycle & 105 & 0.006 \\
\hline & Glucose transport & 36 & 0.031 \\
\hline
\end{tabular}

${ }^{\mathrm{a}} \mathrm{HIV}$, human immunodeficiency virus; ${ }^{\mathrm{b} N E P}$, nuclear export protein; ' $\mathrm{SEP} 2$, septin-2; ${ }^{\mathrm{d} A P O B E C} 3 \mathrm{G}$, apolipoprotein B mRNA-editing, enzymecatalytic, polypeptide-like $3 \mathrm{G} ;{ }^{\mathrm{e}} \mathrm{VIF}$, viral infectivity factor; ${ }_{\mathrm{f}}^{\mathrm{C} D 28}$, custer of differentiation 28 ; ${ }^{\mathrm{g}} \mathrm{BCR}$, B-cell receptor; ${ }^{\mathrm{h}} \mathrm{TCR}$, T-cell receptor; ${ }^{\mathrm{i}} \mathrm{CTLA} 4$, cytotoxic T-lymphocyte antigen 4; ${ }^{\mathrm{T}} \mathrm{TLR}$, toll-like receptor; ${ }^{\mathrm{N}} \mathrm{NLR}$, NOD-like-receptor; ${ }^{\mathrm{PDD}} 1$, programmed death 1 ; ${ }^{\mathrm{m}} \mathrm{CDC} 20$, cell divion cycle protein $20 ;{ }^{\mathrm{n}} \mathrm{CDH} 1$, cadherin-1; ${ }^{\circ} \mathrm{CDC} 6$, cell divion control protein 6 homolog; ${ }^{\mathrm{P} O R C}$, origin recognition complex subunit 1 ; ${ }^{\mathrm{q}} \mathrm{NER}$, nucleotide excision repair; ${ }^{\mathrm{r}} \mathrm{FGFR}$, fibroblast growth factor receptor; ${ }^{\mathrm{s}} \mathrm{SHC}$, Src homomogy 2 domain containing transforming protein; tPI3K, phosphatidylinositol 3- and 4-kinase; "IGFBP, insulin growth factor binding proteins; "TCA, tricarboxylic acid cycle.

Low doses of ionizing radiation induces pro-survival response via immune-stimulation and cell cycle progression responses downstream growth factors signaling. Individual gene pathways analysis showed the downregulation of several growth 


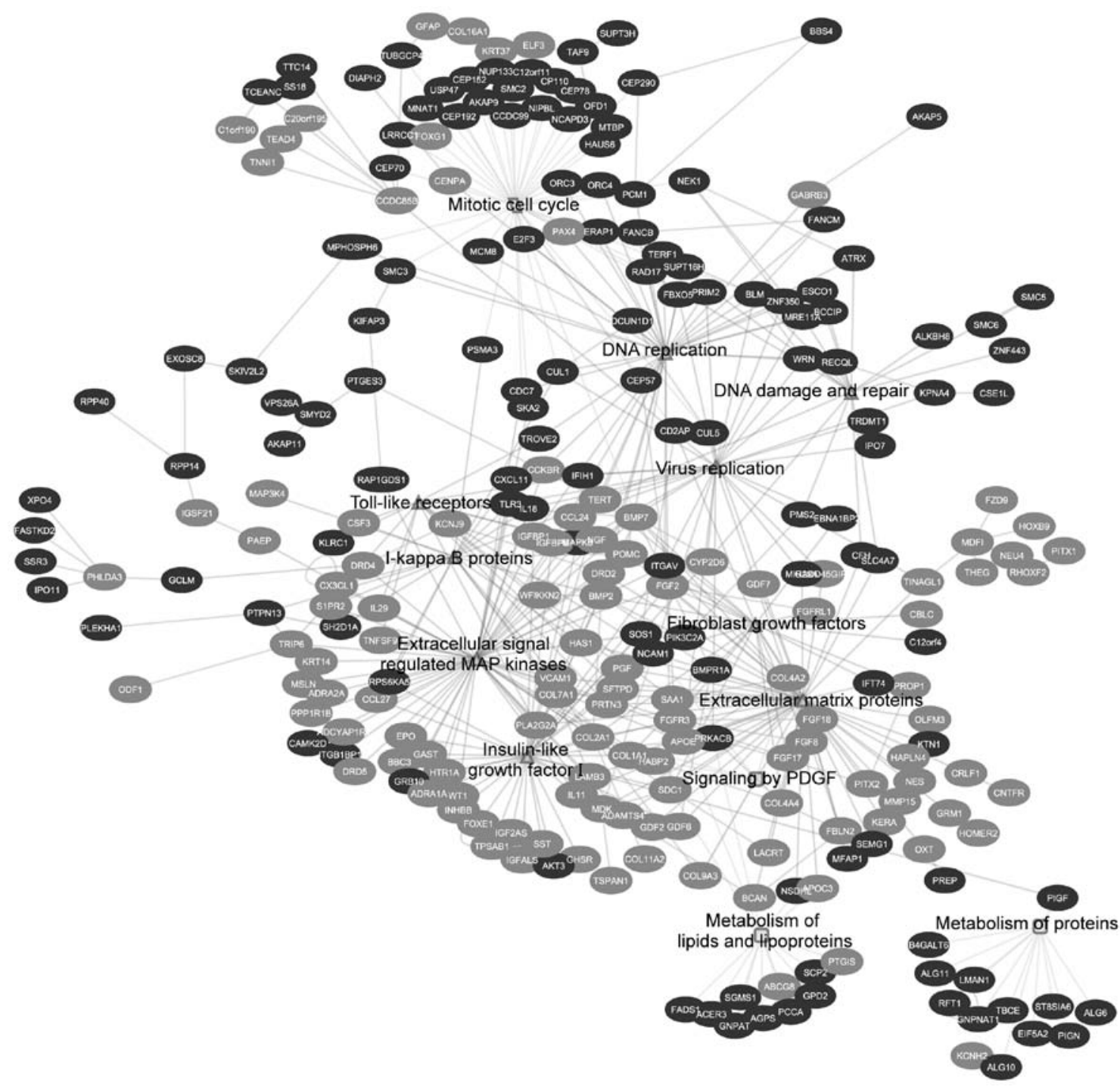

Figure 2. EGAN analysis showing all the differentially expressed enriched pathway genes. Each circle represents a gene. Dark gray circles are upregulated genes; light gray circles are downregulated genes. The lines represent connections between different genes belonging to different pathways.

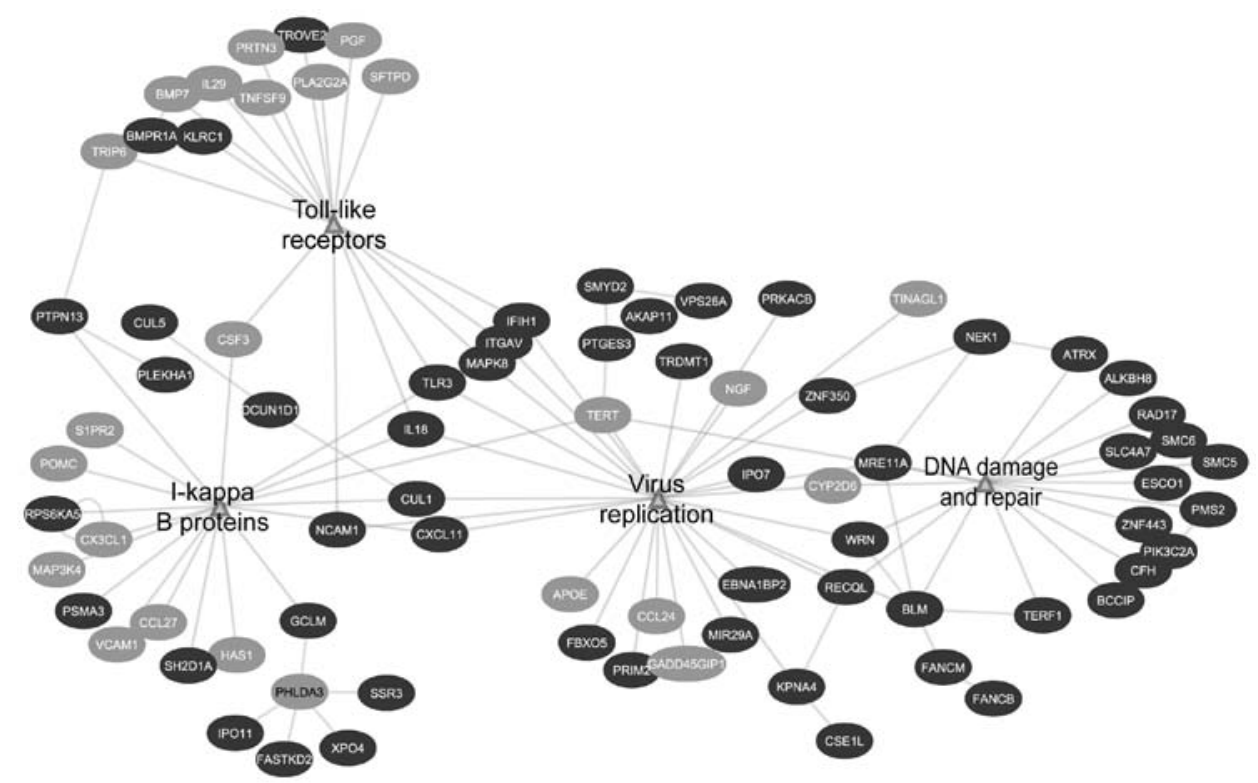

Figure 3. EGAN analysis showing the viral response network. The viral response network is composed from virus replication, IkB proteins, toll-like receptors and DNA damage and repair pathways. Each circle represents a gene. Dark gray circles are upregulated genes; light gray circles are downregulated genes. The lines represent connections between different genes belonging to different pathways. 
Table II. Enriched pathways of the differentially expressed genes.

Pathway p-value

Growth factors signaling and cell cycle

progression

Extracellular matrix proteins

Extracellular signal regulated MAP kinases

Mitotic cell cycle

Insulin growth factor I

Fibroblast growth factors

DNA replication

Signaling by platelet derived growth factor

$1.16 \mathrm{E}-19$

$2.90 \mathrm{E}-16$

$8.70 \mathrm{E}-16$

$2.30 \mathrm{E}-15$

$3.45 \mathrm{E}-11$

$1.98 \mathrm{E}-10$

$2.80 \mathrm{E}-04$

Viral and immune response

Virus replication

2.80E-06

DNA damage and repair

Toll-like receptors

I $\kappa \mathrm{B}$ proteins

$4.90 \mathrm{E}-06$

5.40E-06

$1.90 \mathrm{E}-05$

Metabolism

Metabolism of lipids and lipoproteins

$1.40 \mathrm{E}-05$

Metabolism of proteins

$5.40 \mathrm{E}-04$

RNA degradation
Table III. Number of induced $\gamma-\mathrm{H} 2 \mathrm{AX}$ foci and the equivalent total body dose (ETBD) in the eight prostate cancer patients $30 \mathrm{~min}$ post-IMRT.

\begin{tabular}{llc}
\hline & Induced foci/cell & ETBD (mGy) \\
\hline Patient 1 & 0.622 & 28.01 \\
Patient 2 & 0.584 & 46.34 \\
Patient 3 & 0.267 & 30.24 \\
Patient 4 & 0.674 & 33.79 \\
Patient 5 & 0.583 & 37.94 \\
Patient 6 & 0.561 & 25.54 \\
Patient 7 & 0.28 & 22.07 \\
Patient 8 & 0.194 & 23.86 \\
\hline
\end{tabular}

factors like fibroblast growth factors (FGFs), insulin growth factor I (IGF-I), extracellular proteins and platelet-derived growth factor signaling (PDGF) (Table II). Downstream to growth factor signaling, DNA replication and mitotic cell cycle networks were shown to be upregulated (Fig. 4).

Similarly, GSEA and EGAN showed downregulation of gene sets involved in fibroblast growth factor signaling. These nodes showed a connection with the adaptive immune response gene set and immune-related network, respectively

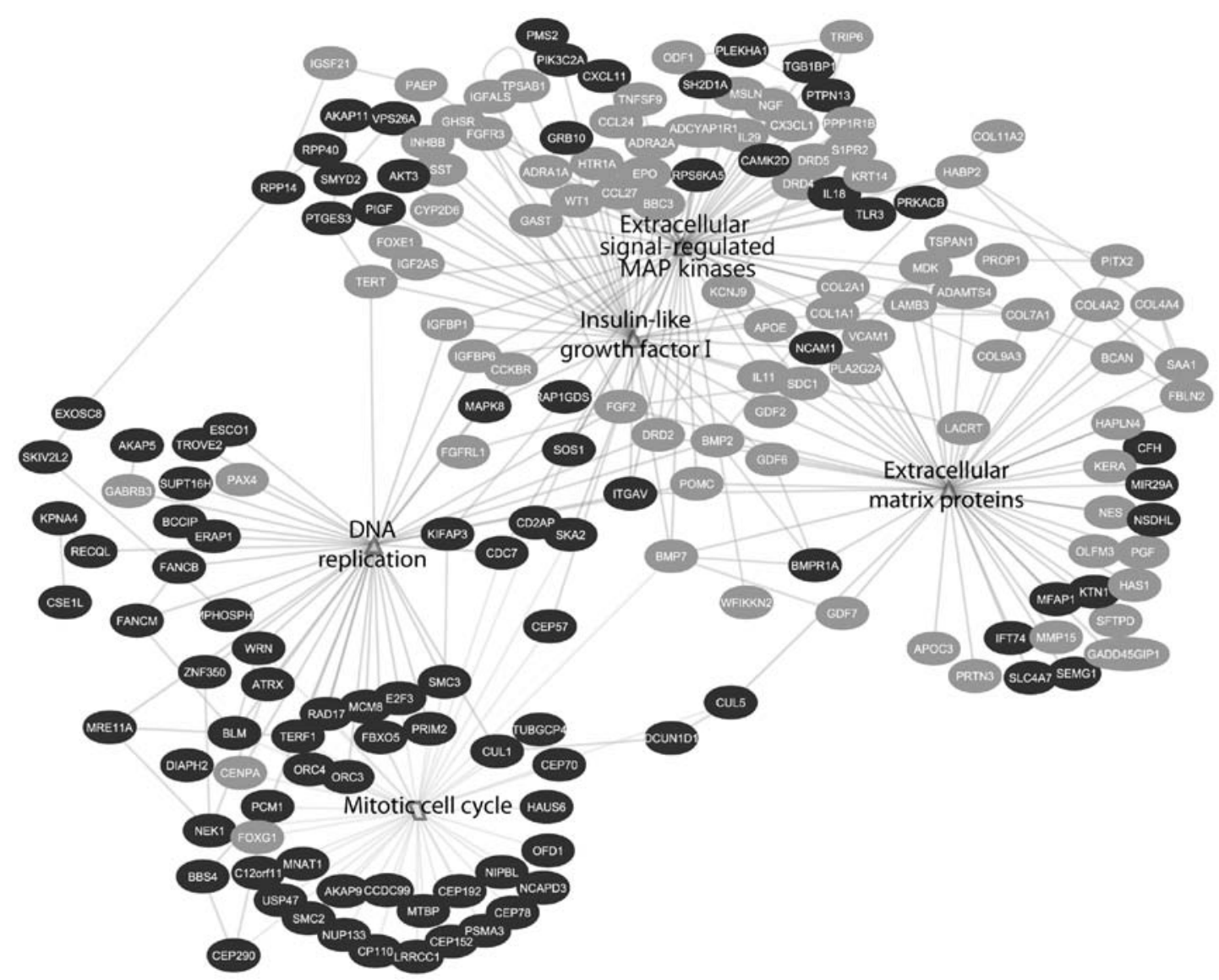

Figure 4. EGAN analysis showing the network between growth factor signaling (fibroblast growth factors, insulin growth factor I, extracellular matrix proteins and ERK MAPKs) and their effect on the positive regulation of cell cycle (mitotic cell cycle and DNA replication). Each circle represents a gene. Dark gray circles are upregulated genes; light gray circles are downregulated genes. The lines represent connections between different genes belonging to different pathways. 


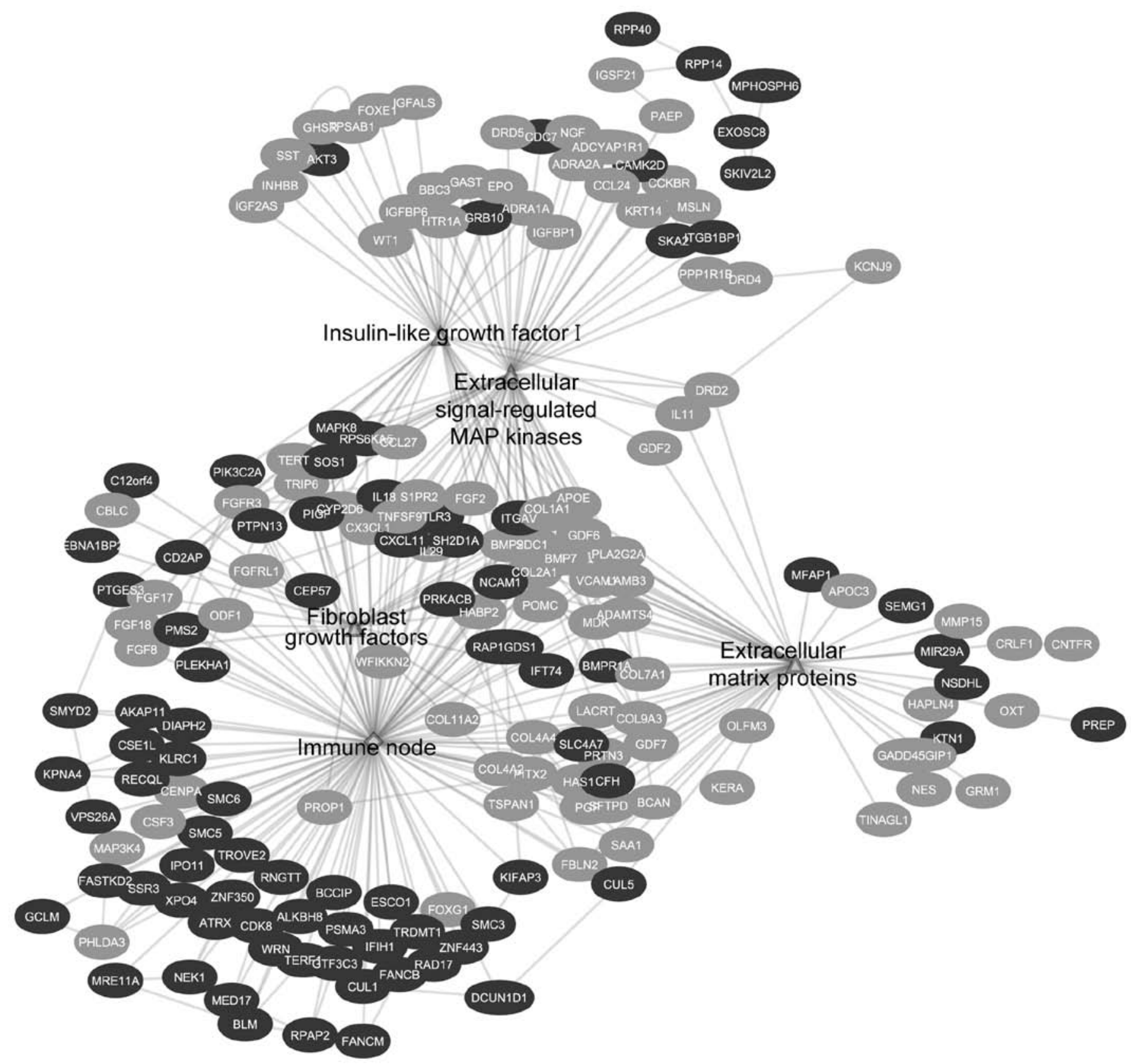

Figure 5. EGAN analysis showing the network between growth factors and their effect on immune-stimulation. The 'Immune node' is the viral response network from Fig. 3. Each circle represents a gene. Dark gray circles are upregulated genes; light gray circles are downregulated genes. The lines represent connections between different genes belonging to different pathways.

(Figs. 1 and 5). Downstream immune-related nodes were connected to the positive cell cycle progression and survival via the induction of the gene sets involved in CDC20, ORC1 and CDH1 degradation and promotion of DNA replication (Figs. 1 and 6). Among the upregulated genes contributing to the positive regulation of cell cycle progression are CCDC 99 , ORC4 and SMC2 (Figs. 4 and 7B).

Low doses of ionizing radiation induces increased DNA damage. For all patients an increase of the $\gamma$-H2AX foci yield was observed: $0.47 \pm 0.19$ foci/cell (Table III). Furthermore, after 18-24 h, significantly upregulated enriched gene sets were determined, and differently expressed genes that are linked to
DNA damage and repair signaling like RAD17, MRE11A, and SMC6 were found (Figs. 3 and 7C).

\section{Discussion}

We investigated in vivo the biological responses to low doses of ionizing radiation. To this end, we assessed DNA damage, through scoring of $\gamma-\mathrm{H} 2 \mathrm{AX}$ foci, and performed whole genome analysis followed by qRT-PCR validation (Fig. 7) on whole blood samples collected from prostate cancer patients undergoing IMRT. Whole blood samples were collected from prostate cancer patients before, and at $30 \mathrm{~min}$ (for $\gamma-\mathrm{H} 2 \mathrm{AX}$ studies), and 18-24 h (for microarray studies) after the first 


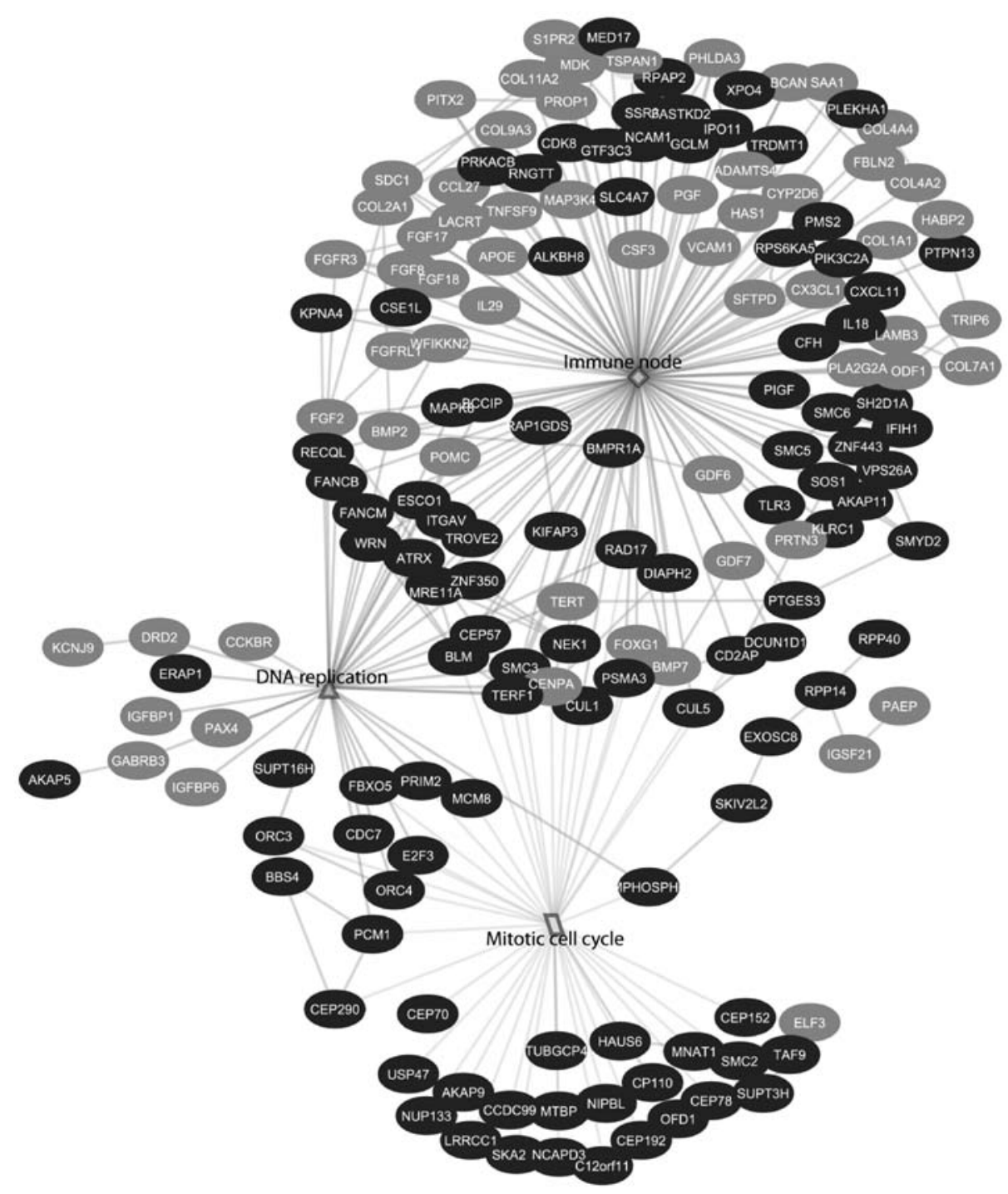

Figure 6. EGAN analysis showing the network between immune-stimulation and their effect on cell cycle progression (mitotic cell cycle and DNA replication). The immune node is the viral response network from Fig. 3. Each circle represents a gene. Dark gray circles are upregulated genes; light gray circles are downregulated genes. The lines represent connections between different genes belonging to different pathways.

fraction of irradiation. We chose to perform the experiments on whole blood samples as these are composed of a complex combination of different cell types; therefore, it allows the study of a collective tissue response. On the other hand, blood is a circulating tissue, thus it reflects the response to the calculated equivalent total body dose.

Prostate cancer patients show induction of pro-inflammatory response via the activation of viral signaling. Previously, we demonstrated that low doses of ionizing radiation induce a unique gene expression profile compared to high doses (11). The low doses are characterized by the induction of stimulatory immune response through the activation of chemokine and cytokine signaling, while high doses are characterized by a damaging response through p53 signaling. In agreement with these results, current GSEA showed the enrichment of several immune signaling pathways; top ranked gene sets were related to viral signaling, in specific human immunodeficiency virus (HIV) infection signaling and interferon secretion (Table I). Viral response is composed of signaling network between
NF-kB, ERK 1/2 MAP kinase and p38 MAP kinase pathways. Furthermore, it is known that ionizing radiation is able to activate HIV promoter and gene expression in T cells. The gene expression of HIV viral infections are regulated by various cell signaling events that combine mitogens, cytokines, stress, and DNA damage (18). In other words, the enrichment of the HIV-infection and interferon gene sets in our data suggests a 'communication network' between DNA damage and central pathways in the immune response (Figs. 1 and 3). In addition, to that, other viral-related gene sets were also shown to be upregulated; these are NEP/SEP viral proteins, subset of the HIV-infection gene set, and degradation of APOBEC3G via VIF (viral infectivity factor). APOBEC $3 \mathrm{G}$ is a protein that plays a role in activating an antiviral response; its degradation denotes an amplification of viral and inflammatory response (19). One of the key genes that plays a role in response to viral infections is the toll-like receptor 3 (TLR3) (Fig. 7A), after viral infection TLR3 recognizes double strand RNA (dsRNA) that leads to downstream activation of type I interferons and $\mathrm{NF}-\kappa \mathrm{B}$, a proinflammatory and prosurvival pathway $(20,21)$. 

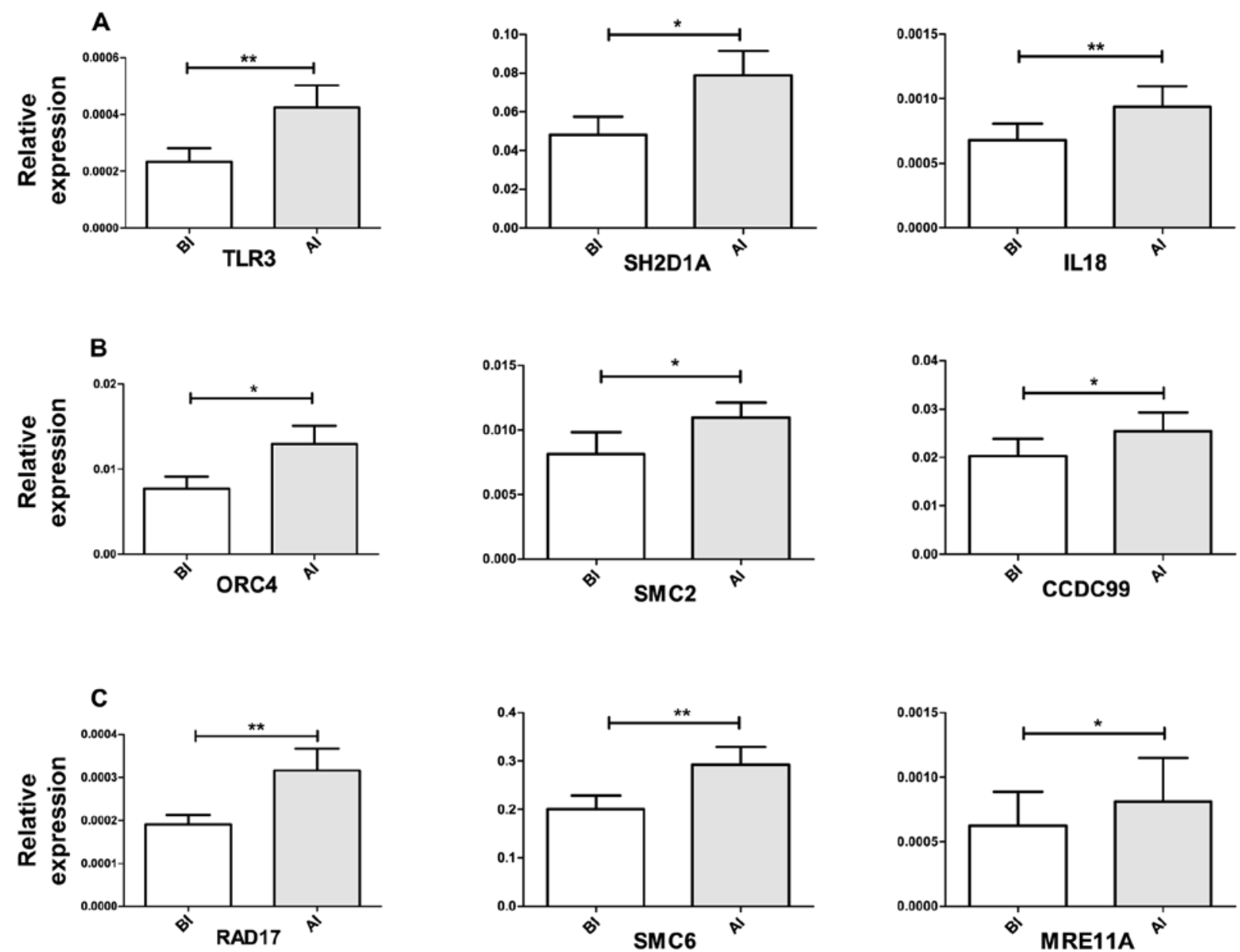

Figure 7. Comparative quantitative RT-PCR validation on genes differentially expressed. (A) Pro-inflammatory response stimulation (TLR3, SH2D1A and IL18); (B) cell cycle progression (ORC4, SMC2 and CCDC99); (C) DNA damage and repair (RAD17, SMC6 and MRE11A). Relative expression levels were calculated using Pffafl method normalized to $P G K 1$ gene levels. Statistical comparison on the level of induction between the control and irradiated samples was done by applying paired t-test. A $\mathrm{p}<0.05$ was considered as significant difference between the two conditions. ${ }^{*} \mathrm{p}<0.05,{ }^{* *} \mathrm{p}<0.005,{ }^{* * *} \mathrm{p}<0.0001$. BI, before irradiation; AI, after irradiation.

TLR3 was reported also to be activated upon interaction with exogenous and endogenous RNA molecules (22). Furthermore, GSEA showed the enrichment of TLR cascade gene set (Table I), where TLR3, 7 and 8, involved in viral signaling, contributed to the enrichment score (23). In addition, several genes playing a role in viral signaling and interferon induction such as IFIHI, MAPK8, KPNA4 and ILI8 genes were shown to be upregulated (Fig. 3).

Downstream of the activation of TLRs and interferons is the NF- $\mathrm{BB}$ signaling pathway, where EGAN analysis showed deregulation of I $\mathrm{B}$ proteins (Table II). Overexpression of SH2DIA and IL18 (Fig. 7A), and CUL1 may indicate the positive regulation of NF- $\mathrm{KB}$ signaling (Fig. 3) (24-26).

Prostate cancer patients show induction of pro-inflammatory response via the activation of adaptive and innate immune signaling. Previously, we have demonstrated that low doses induce the activation of $\mathrm{T}$ - and $\mathrm{B}$-cell receptors and innaterelated gene set, such as toll-like receptors, NOD-like receptors and RIG-like receptors (11). In agreement with these results, GSEA showed the enrichment of several gene sets that are involved in the stimulation of the immune response via the activation of both adaptive and innate immune responses. The second ranked immune gene set was CD28 stimulation, which is related also to the CTLA4 inhibition gene set (Table I). $\mathrm{T}$ cell activation is dependent on the opposing signaling from two cell receptors CD28 and CTL4A. Liu and colleagues (27) have reported that stimulation of CD28 is dose-dependent and specific to low doses of ionizing radiation. Furthermore, the same group reported upregulation in CD28 and downregulation of CTLA4 in lymphocytes isolated from mouse blood exposed to $0.075 \mathrm{~Gy}$ whole body irradiation. They showed also that the interaction between antigen presenting cells and $\mathrm{T}$ cells is suppressed after exposure of mice to 2-Gy whole body irradiation as a result of CTLA4 upregulation (28). In addition, programmed death 1 (PD1) signaling was shown to be upregulated; PD1 is a surface membrane protein that plays a role in attenuating autoimmune responses, thus it acts in response to the increased activity of the T cell signaling (29). Other gene sets related to innate immune response and inflammation were shown to be activated as well; these include phagosome pathway and inflammasome formation.

Prostate cancer patients show induction of pro-survival response via immune-stimulation and cell cycle progression responses downstream the growth factor signaling. There 
is growing evidence that low doses of ionizing radiation have a proliferative and pro-survival responses through the involvement of growth factors (11,30-32). Our data showed downregulation in several growth factors pathways, e.g. FGF, IGF-I and PDGF and several molecules involved in extracellular matrix (ECM) molecules (e.g. LAMB3, COL2OA1 and $C O L 9 A 3)$ that are involved in growth signaling. This could be related to the late time-point. GSEA and EGAN analysis showed that the growth factor signaling cluster showed a connection with the adaptive immune response node and the viral response node; growth factors, such as FGF were previously shown to be involved in an immune-stimulatory reaction in response to lipopolysaccharide (LPS) stimulation $(33,34)$. Furthermore, genes playing a role in the positive regulation of ERK, MAPK and NF- $\kappa \mathrm{B}$ signaling were shown to be upregulated, such as the induction of SOS1, ITGAV, AKT3, PIK3C2A, $M A P K 8, S H 2 D 1 A$ and ILI8 (Figs. 3 and 5).

Furthermore, both analyses showed that viral and immune response gene sets were connected to the nodes of the cell cycle progression and DNA replication (Figs. 1, 6 and 7C). Previously, it was reported that regulation of cell cycle is a characteristic of a low dose response $24 \mathrm{~h}$ post-irradiation $(9,10)$. In contrast to our expectation, cell cycle was not arrested and cell cycle checkpoints were not activated, probably due to the low doses received by the patients and the cell cycle positive regulation of the downstream growth factor and immune stimulation.

Prostate cancer patients show increased DNA damage and anti-apoptotic response post-IMRT. DNA damage signaling was induced 30 min post-irradiation (Table III) and did not terminate 18-24 h later (Figs. 3 and 7B). Taking into account that the cell cycle arrest was not activated (Fig. 4), and was shown not to be launched under a threshold of $200 \mathrm{mGy}$; this might increase the possibility of carrying unrepaired or misrepaired DNA breaks through the cell division process, thus induction of cancers would be more probable $(35,36)$. In addition, p53 signaling, which is known to be a central player in response to ionizing radiation (37), was not enriched in either analysis approach. The DNA damage and repair response was accompanied by an anti-apoptotic response; where genes involved in stabilization of p53 were downregulated (PHLDA3) (38) while others involved in its degradation were upregulated (MTBP) (39). BBC3, belongs to the $\mathrm{BH} 3$-only pro-apoptotic genes, and was also downregulated.

In conclusion, our study demonstrated that immunestimulatory signaling played a central role in response to low doses of ionizing radiation. These results are in agreement with those reported previously in our in vitro whole genome analysis (11). Furthermore, we showed that responses to low doses are a communication network between growth factors and cell cycle progression pathways stimulated by immune signaling. Moreover, we report that remaining unrepaired DNA damage still exists after 18-24 h.

Our study addresses the need for reconsideration of the health risks from the out-of-field low doses of ionizing radiation exposed to the normal tissues when undergoing IMRT. Inflammatory and DNA damage responses may carry the risk of development of systematic inflammations and secondary cancers, respectively; there is accumulating number of studies that show advantages of using particle therapy over treatments that use X-rays. It is demonstrated that the healthy surrounding tissues are spared from out-field radiation. However, other studies have reported that secondary neutrons can carry the risk of developing secondary cancers during particle therapy. There are still no clear-cut answers for a 'perfect' radiotherapy approach, and further inter-disciplinary research is still required $(7,40)$.

\section{Acknowledgements}

The authors are thankful to Ghent University Hospital patients who kindly accepted to participate in this study. Also, we appreciate Dr P. Willems [Federal Agency for Nuclear Control (FANC), Belgium] for the fruitful scientific discussions carried out through the preparation of the study. H. El-Saghire was supported by a doctoral SCK·CEN/Ghent University grant. This study was funded by the FANC CT-SCAN contract (CO-90-09-2329-00) and by the FP7 EU EPI-CT contract (grant agreement 269912).

\section{References}

1. Ahmad SS, Duke S, Jena R, Williams MV and Burnet NG: Advances in radiotherapy. BMJ 345: e7765, 2012.

2. Hall EJ and Wuu CS: Radiation-induced second cancers: the impact of 3D-CRT and IMRT. Int J Radiat Oncol Biol Phys 56: 83-88, 2003.

3. Purdy JA: Dose to normal tissues outside the radiation therapy patient's treated volume: a review of different radiation therapy techniques. Health Phys 95: 666-676, 2008.

4. Hall EJ: Intensity-modulated radiation therapy, protons, and the risk of second cancers. Int J Radiat Oncol Biol Phys 65: 1-7, 2006.

5. Ruben JD, Davis S, Evans C, et al: The effect of intensity-modulated radiotherapy on radiation-induced second malignancies. Int J Radiat Oncol Biol Phys 70: 1530-1536, 2008.

6. Ost P, Speleers B, De Meerleer G, et al: Volumetric arc therapy and intensity-modulated radiotherapy for primary prostate radiotherapy with simultaneous integrated boost to intraprostatic lesion with 6 and $18 \mathrm{MV}$ : a planning comparison study. Int J Radiat Oncol Biol Phys 79: 920-926, 2011.

7. Murray L, Henry A, Hoskin P, Siebert FA and Venselaar J; BRAPHYQS/PROBATE group of the GEC ESTRO: Second primary cancers after radiation for prostate cancer: a review of data from planning studies. Radiat Oncol 8: 172, 2013.

8. Wyrobek AJ, Manohar CF, Krishnan VV, et al: Low dose radiation response curves, networks and pathways in human lymphoblastoid cells exposed from 1 to $10 \mathrm{cGy}$ of acute gamma radiation. Mutat Res 722: 119-130, 2011.

9. Yunis R, Albrecht H, Kalanetra KM, Wu S and Rocke DM: Genomic characterization of a three-dimensional skin model following exposure to ionizing radiation. J Radiat Res 53: 860-875, 2012.

10. Ray M, Yunis R, Chen X and Rocke DM: Comparison of low and high dose ionising radiation using topological analysis of gene coexpression networks. BMC Genomics 13: 190, 2012.

11. El-Saghire H, Thierens H, Monsieurs P, Michaux A, Vandevoorde $\mathrm{C}$ and Baatout S: Gene set enrichment analysis highlights different gene expression profiles in whole blood samples X-irradiated with low and high doses. Int J Radiat Biol 89: 628-638, 2013.

12. Pernot E, Hall J, Baatout $\mathrm{S}$, et al: Ionizing radiation biomarkers for potential use in epidemiological studies. Mutat Res 751: 258-286, 2012.

13. Subramanian A, Tamayo P, Mootha VK, et al: Gene set enrichment analysis: a knowledge-based approach for interpreting genome-wide expression profiles. Proc Natl Acad Sci USA 102: 15545-15550, 2005.

14. Werbrouck J, De Ruyck K, Beels L, et al: Prediction of late normal tissue complications in RT treated gynaecological cancer patients: potential of the gamma-H2AX foci assay and association with chromosomal radiosensitivity. Oncol Rep 23: 571-578, 2010. 
15. Irizarry RA, Hobbs B, Collin F, et al: Exploration, normalization, and summaries of high density oligonucleotide array probe level data. Biostatistics 4: 249-264, 2003.

16. Merico D, Isserlin R, Stueker O, Emili A and Bader GD Enrichment map: a network-based method for gene-set enrichment visualization and interpretation. PLoS One 5: e13984, 2010.

17. Pfaffl MW: A new mathematical model for relative quantification in real-time RT-PCR. Nucleic Acids Res 29: e45, 2001.

18. Oakley JD, Taher MM, Hershey CM, Aggarwal PC, Estwani IB and Valerie K: Triggering of apoptosis is not sufficient to induce human immunodeficiency virus gene expression. IUBMB Life 55: 415-427, 2003.

19. Nowarski R, Wilner OI, Cheshin O, et al: APOBEC3G enhances lymphoma cell radioresistance by promoting cytidine deaminase-dependent DNA repair. Blood 120: 366-375, 2012.

20. Zhu J, Ghosh A, Coyle EM, et al: Differential effects of phenethyl isothiocyanate and D,L-sulforaphane on TLR3 signaling. J Immunol 190: 4400-4407, 2013.

21. Amarante MK and Watanabe MA: Toll-like receptor 3: involvement with exogenous and endogenous RNA. Int Rev Immunol 29: 557-573, 2010.

22. Bauernfeind F, Ablasser A, Kim S, Bartok E and Hornung V: An unexpected role for RNA in the recognition of DNA by the innate immune system. RNA Biol 7: 151-157, 2010.

23. Vercammen E, Staal J and Beyaert R: Sensing of viral infection and activation of innate immunity by toll-like receptor 3 . Clin Microbiol Rev 21: 13-25, 2008.

24. Chuang HC, Wang JM, Hsieh WC, Chang Y and Su IJ: Up-regulation of activating transcription factor-5 suppresses SAP expression to activate $\mathrm{T}$ cells in hemophagocytic syndrome associated with Epstein-Barr virus infection and immune disorders Am J Pathol 173: 1397-1405, 2008.

25. Surjit M, Varshney B and Lal SK: The ORF2 glycoprotein of hepatitis E virus inhibits cellular NF-kappaB activity by blocking ubiquitination mediated proteasomal degradation of IkappaBalpha in human hepatoma cells. BMC Biochem 13: 7 , 2012

26. Hayden MS and Ghosh S: NF-kappaB, the first quarter-century: remarkable progress and outstanding questions. Genes Dev 26: 203-234, 2012.

27. Liu SZ, Jin SZ, Liu XD and Sun YM: Role of CD28/B7 costimulation and IL-12/IL-10 interaction in the radiation-induced immune changes. BMC Immunol 2: 8, 2001.
28. Shan YX, Jin SZ, Liu XD, Liu Y and Liu SZ: Ionizing radiation stimulates secretion of pro-inflammatory cytokines: doseresponse relationship, mechanisms and implications. Radiat Environ Biophys 46: 21-29, 2007.

29. Riley JL: PD-1 signaling in primary T cells. Immunol Rev 229 114-125, 2009.

30. Kim SJ, Dix DJ, Thompson KE, et al: Effects of storage, RNA extraction, genechip type, and donor sex on gene expression profiling of human whole blood. Clin Chem 53: 1038-1045, 2007.

31. Liang X, So YH, Cui J, et al: The low-dose ionizing radiation stimulates cell proliferation via activation of the MAPK/ERK pathway in rat cultured mesenchymal stem cells. J Radiat Res 52. 380-386, 2011.

32. Sofia Vala I, Martins LR, Imaizumi N, et al: Low doses of ionizing radiation promote tumor growth and metastasis by enhancing angiogenesis. PLoS One 5: e11222, 2010.

33. Marcinkowska E, Superat K and Wiedlocha A: FGF-1 as a possible carrier for targeted drug delivery. Oncol Res 16: 27-34, 2006.

34. Shi M, Lin TH, Appell KC and Berg LJ: Cell cycle progression following naive $\mathrm{T}$ cell activation is independent of Jak3/common gamma-chain cytokine signals. J Immunol 183: 4493-4501, 2009.

35. Fernet M, Megnin-Chanet F, Hall J and Favaudon V: Control of the $\mathrm{G} 2 / \mathrm{M}$ checkpoints after exposure to low doses of ionising radiation: implications for hyper-radiosensitivity. DNA Repair 9: 48-57, 2010

36. Wood ME, Vogel V, Ng A, Foxhall L, Goodwin P and Travis LB: Second malignant neoplasms: assessment and strategies for risk reduction. J Clin Oncol 30: 3734-3745, 2012.

37. Rashi-Elkeles S, Elkon R, Shavit S, et al: Transcriptional modulation induced by ionizing radiation: p53 remains a central player. Mol Oncol 5: 336-348, 2011.

38. Aviv Y and Kirshenbaum LA: Novel phosphatase PHLPP-1 regulates mitochondrial Akt activity and cardiac cell survival. Circ Res 107: 448-450, 2010

39. Alam MJ, Fatima N, Devi GR, Ravins and Singh RK: The enhancement of stability of p53 in MTBP induced p53-MDM2 regulatory network. Biosystems 110: 74-83, 2012.

40. Newhauser WD and Durante M: Assessing the risk of second malignancies after modern radiotherapy. Nat Rev Cancer 11: 438-448, 2011. 